\title{
Perfil clínico dos pacientes no atendimento médico em uma emergência cardiológica de referência no estado de Pernambuco
}

\author{
Mariana Barros Mendonça Figueirêdo 1, *, Natália de Castro Borges 1, Manuela Barbosa Rodrigues de \\ Souza 2, Pedro Rafael Salerno 2
}

${ }^{1}$ Discente em Medicina da Universidade Católica de Pernambuco (UNICAP).

${ }^{2}$ Docente do Centro de Ciências Biológicas e da Saúde da Universidade Católica de Pernambuco (UNICAP).

*Corresponding author: Mariana Barros Mendonça Figueiredo. Rua do Príncipe, $n^{0} 526$ - Boa Vista. Zip Code: 50050-900 - Pernambuco, PE, Brazil. E-mail: maribmfigueiredo@gmail.com.

Research Ethics Committee Approval (if necessary): Approved by the Universidade Católica de Pernambuco Ethics Committee (\#3.425.265).

Received on: Jul 31, 2021. Accepted on: Aug 7, 2021. Available online: Aug 20, 2021.

\begin{abstract}
O estudo objetivou avaliar o perfil clínico que propicia a procura de um atendimento no serviço de emergência cardiológica do Pronto-Socorro Cardiológico Universitário de Pernambuco Prof ${ }^{\circ}$ Luiz Tavares (PROCAPE). Trata-se de um estudo descritivo, transversal, quantitativo com aplicação de um questionário a 104 pacientes atendidos na emergência cardiológica do PROCAPE de setembro a novembro de 2019, com posterior análise de seus prontuários, para determinar se o diagnóstico médico é uma emergência cardiológica, ou outra, e se os pacientes tinham histórico de doença cardiológica. No estudo, $61,6 \%$ têm de 50 a 70 anos, $82,7 \%$ são do sexo masculino e $77,3 \%$ são hipertensos. Quanto ao tempo de início dos sintomas, $36,5 \%$ os apresentaram em minutos/horas, enquanto 63,5\% tinham há dias/meses. Pelo prontuário, 81 eram uma emergência cardiológica, 04 emergências não cardiológica, 19 eram ambulatoriais. No questionário, 60,6\% disseram ter diagnóstico prévio de doença cardiovascular, enquanto 39,4\% responderam não ter. Porém, no prontuário, desses 39,4\%, 29,8\% tinham cardiopatia prévia e não sabiam, enquanto $9,6 \%$ de fato não tinha o histórico. Logo, o perfil clínico dos usuários é em sua maioria indivíduos do sexo masculino, adultos e idosos, portadores de emergência cardiológica, cuja maioria possui cardiopatia clínica prévia.
\end{abstract}

Palavras-chaves: Adulto; Emergência; Diagnóstico; Registros Médicos.

\section{Introdução}

A unidade de serviço de emergência consiste em um dos pilares do funcionamento do sistema de saúde que atende os pacientes que chegam em estado grave, assim como acolhe os não urgentes e encaminha para serviços 
ambulatoriais ou especializados que compõe a rede de saúde. Em compensação, existe um excessivo volume de atendimento pela demanda da população não esperado pela gestão hospitalar que ultrapassa os limites para um atendimento mais humanizado, provocando sofrimento, baixa qualidade de atendimento e sequelas ao paciente [1].

Não há uma definição universal específica de uma visita não urgente ao departamento de emergência [2]. Na maioria das vezes, os pacientes categorizados como não-urgentes são definidos como aqueles que poderiam ter sido tratados por um clínico geral. Os principais motivos que levam os pacientes não-urgentes a procurarem um serviço de saúde de emergência são: atender às necessidades de saúde como dor, desconforto e ansiedade pela queixa; ultrapassar as barreiras do acesso primário à saúde; vantagens da disponibilidade de recursos, incluindo exames laboratoriais e de imagem.

O perfil demográfico do Brasil vem mostrando um aumento significativo do número de idosos e um aumento da população simultâneo ao surgimento de doenças crônicas não transmissíveis (DCNT) que colaboram para o aumento dos custos e suporte das unidades de saúde. No cenário epidemiológico, destacam-se as doenças cardiovasculares que atingem expressiva parcela da população sendo frequente causa de internação hospitalar, tendo impacto direto sobre a organização da atenção a saúde SUS [3].

Segundo a World Health Organization [4], as doenças cardiovasculares são a principal causa de morte no mundo, sendo o número de mortes no mundo devidas às doenças cardiovasculares foi de 17,3 milhões em 2008, o que representa 30,5\% do total de óbitos nesse ano. No Brasil, elas foram responsáveis por $28,6 \%$ das mortes, totalizando 335.213 óbitos em 2011. Desses óbitos, 103.486 foram devidos às doenças arteriais coronarianas (DAC), correspondendo a $8,8 \%$ dos óbitos no país em 2011. No estado de Pernambuco, no ano de 2008 houveram 16.656 óbitos relacionados a doenças do aparelho circulatório em ambos os sexos com faixa etária acima de 20 anos. Petrolina no mesmo ano apresentou 319 óbitos relacionados a cardiopatias [5].

Dentro da prevenção, o monitoramento da prevalência dos fatores de risco para DCV, especialmente os de natureza comportamental, permitem, por meio das evidências observadas, a implementação de ações preventivas com maior custo-efetividade. Assim, um maior investimento na identificação precoce das DCNT e seu controle na atenção primária permite uma menor lotação das emergências e diminuição de mortes, principalmente naqueles pacientes com histórico de agudização de doença previamente estabelecida [1].

O objetivo do presente estudo foi estratificar o perfil clínico dos pacientes 
no atendimento médico da emergência cardiológica do Pronto-Socorro Cardiológico Universitário de Pernambuco Prof ${ }^{\circ}$ Luiz Tavares (PROCAPE).

\section{Metodologia}

Estudo transversal, descritivo e quantitativo, com a aplicação de um questionário (Anexo) pelos pesquisadores, a pacientes que procuraram atendimento médico na emergência cardiológica do ProntoSocorro Cardiológico Universitário de Pernambuco Prof ${ }^{\circ}$ Luiz Tavares (PROCAPE) durante o período de setembro a novembro de 2019. Além disso, foram avaliados os prontuários eletrônicos dos mesmos pacientes no período de dezembro de 2019 a janeiro de 2020 para analisar se o diagnóstico médico é uma emergência cardiológica, uma emergência não cardiológica ou de causa ambulatorial cardiológica ou não cardiológica. Também foram comparados os resultados em pacientes com histórico de doença cardiológica aos que não têm histórico.

Foram incluídos pacientes a partir da faixa etária dos 18 anos que procuraram atendimento médico na emergência cardiológica do PROCAPE, sendo descartados pacientes em estado crítico com impossibilidade de responder ao questionário, pacientes sem acompanhantes que possam responder por completo o questionário, os que se neguem a participar da pesquisa e pacientes que não constam com informações no prontuário.

O questionário foi elaborado pelos pesquisadores, e contém 15 perguntas, compostas por três domínios. Foram utilizadas as perguntas do terceiro sobre caracterização de emergência cardiológica, da pergunta 12 a 15.

Após o levantamento dos dados, foi realizada a interpretação estatística de cada variável analisada através dos questionários e prontuários, e os resultados estão apresentados sob a forma de gráficos e/ou tabelas com suas respectivas frequências absoluta $\mathrm{e}$ relativa, produzidos em planilhas do Excel (Windows 2010) e Softwares STATA/SE 12.0, que evidenciaram a prevalência de cada variável em relação à amostra. Todos os testes foram aplicados com $95 \%$ de confiança.

Todos os aspectos éticos foram contemplados por meio da garantia da privacidade dos dados obtidos, onde a sua utilização será apenas e exclusivamente para fins científicos. $\mathrm{O}$ projeto foi submetido à instituição coparticipante, a emergência cardiológica de referência do estado de Pernambuco (Pronto-Socorro Cardiológico Universitário de Pernambuco Prof ${ }^{\circ}$ Luiz Tavares (PROCAPE), e ao Comitê de Ética em Pesquisa da Universidade Católica de Pernambuco, sob parecer $n^{\circ}$ 3.425.265. 


\section{Resultados}

Participaram do estudo 106 usuários do serviço de emergência do PROCAPE, dos quais 104 se adequaram aos critérios de inclusão e exclusão, sendo, portanto, analisadas 104 entrevistas e prontuários. Mais da metade está na faixa etária dos 50 aos 70 anos $(61,6 \%)$, e a maioria, 86 pacientes $(82,7 \%)$, são do sexo masculino (Tabela $1)$.

Table 1. Características sociodemográficas dos atendidos na emergência do PROCAPE.

\begin{tabular}{lcc}
\hline \multicolumn{1}{c}{ Variáveis } & N (104) & \% \\
\hline Idade & & \\
$<50$ anos & 15 & 14,4 \\
$50-70$ anos & 64 & 61,6 \\
$>70$ anos & 25 & 24,0 \\
Sexo & & \\
Masculino & 86 & 82,7 \\
Feminino & 18 & 17,3 \\
\hline
\end{tabular}

Considerando o motivo de busca pelo atendimento ao serviço de emergência do PROCAPE, dos quais um mesmo paciente poderia ter mais de um motivo, observou-se que dor no peito $(72,1 \%)$, dispneia $(65,4 \%)$ e fadiga $(55,8 \%)$ são os principais sintomas relatados na porta de entrada do serviço. Apenas dois pacientes (1,9\%) entraram na emergência assintomáticos, um deles para realizar exame de ecocardiografia por histórico de angina e infarto agudo do miocárdio (IAM), enquanto o outro teve diagnóstico de IAM silencioso (Gráfico 1).

Gráfico 1. Sintomas que justificam o motivo de busca pelo atendimento no serviço de emergência do PROCAPE.

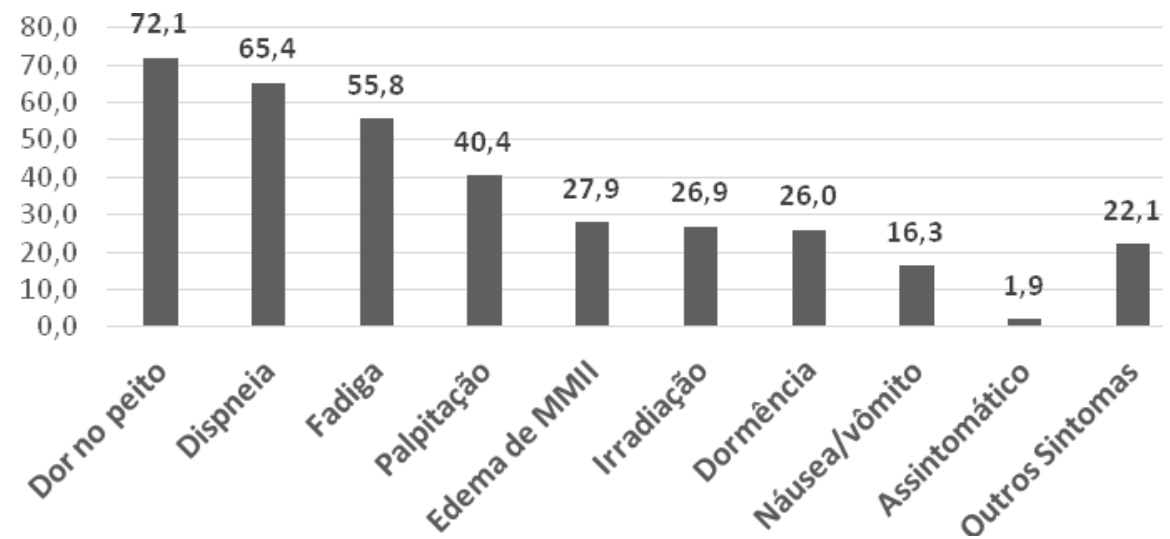


Ao estratificar os atendimentos na emergência cardiológica a partir da análise de prontuários, observou-se que $81(77,9 \%)$ eram de fato para estarem lá por se constar de uma emergência cardiológica, contudo dos 104, 04 eram uma emergência não cardiológica, 18 apresentaram queixas que poderiam ser tratadas no ambulatório cardiológico, e apenas 01 apresentou uma queixa ambulatorial não cardiológica (Tabela 2).

Tabela 2: Caracterização do tipo de atendimento prestado aos pacientes de acordo com a patologia de base ao chegarem na emergência cardiológica do PROCAPE avaliado pelo prontuário.

\section{Variáveis}

$$
\mathrm{n}=104
$$

$\%$

\section{Tipo de Atendimento}

Emergência cardiológica

Emergência não

4

cardiológica

Ambulatório cardiológico

Ambulatório não

cardiológico

Em se tratando de doença cardiovascular prévia, ao comparar as respostas do questionário com as informações do prontuário eletrônico, observou-se que dos 41 (39,4\%) que negaram cardiopatia prévia no questionário, $31 \quad(29,8 \%) \quad$ tinham diagnóstico de cardiopatia prévia pelo prontuário, enquanto $10(9,6 \%)$ de fato não tinham o histórico, e dos $63(60,6 \%)$ que responderam que tinham histórico, todos obtiveram confirmação dele pelo prontuário (Gráfico 2).
Entre

as

doenças cardiovasculares prévias, e sabendo que um mesmo paciente pode ter mais de um diagnóstico, 77,3\% dos indivíduos da emergência eram hipertensos e 38,1\% tiveram episódio de infarto agudo do miocárdio (IAM) prévio. As demais doenças mantiveram uma equivalência de porcentagem, variando de 9,3\% a $11,3 \%$, enquanto apenas $3,1 \%$ não obtinham diagnóstico (Gráfico 3). 
Gráfico 2: Quantificação de pacientes com e sem histórico de doença cardiovascular prévio ao analisar o prontuário.
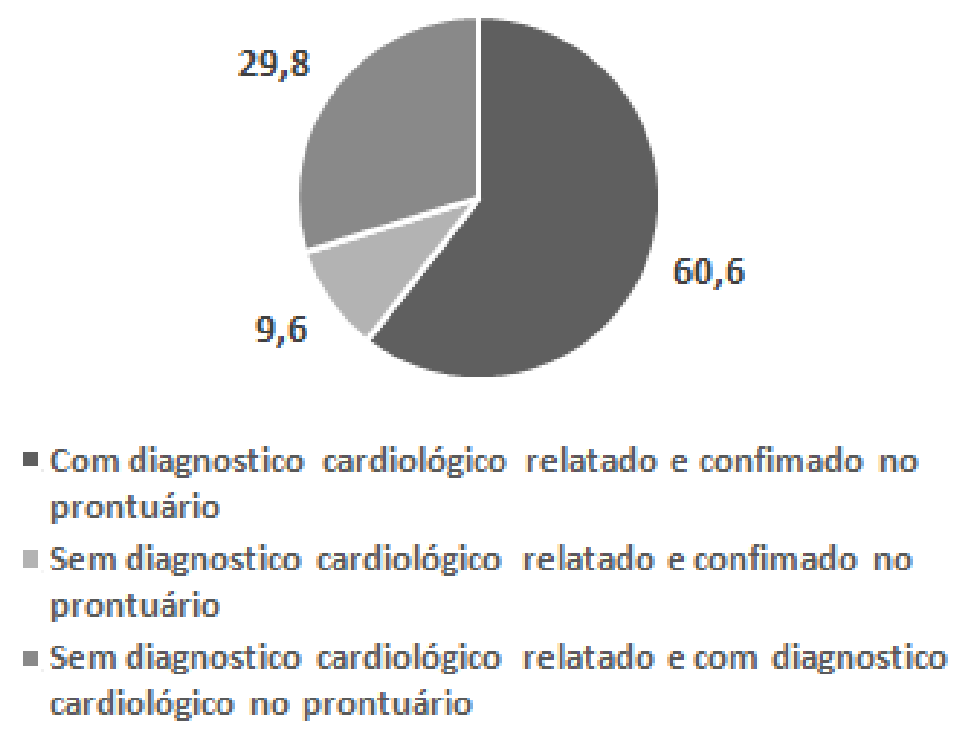

Gráfico 3: Doenças cardiológicas com diagnóstico prévias confirmadas pelo prontuário em pacientes atendidos pela emergência do PROCAPE.

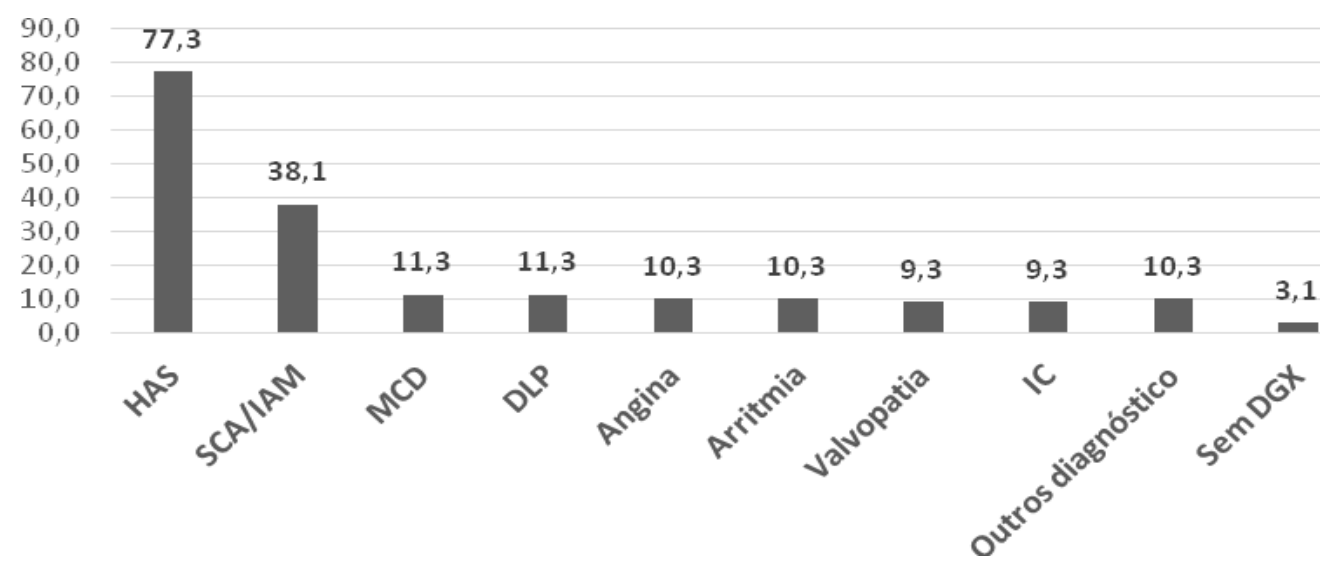

Legenda: HAS: hipertensão arterial sistêmica; SCA/IAM: síndrome coronariana aguda/infarto agudo do miocárdio; MCD: miocardiopatia dilatada; DLP: dislipidemia; IC: insuficiência cardíaca; Sem DGX: sem diagnóstico.

\section{Discussão e Conclusão}

O conhecido estudo de Framingham [6] tem fornecido uma visão sobre os fatores de risco envolvidos na etiologia da doença cardiovascular aterosclerótica, sendo a idade avançada um preditor de risco. A tabela 1 evidencia que o perfil sociodemográfico da amostra foi similar 
conforme os estudos [7], [8] e [9], revelando, principalmente, idade média de 60 anos, com ligeira predominância entre o sexo masculino, porém divergente quanto ao sexo nos estudos [10], [11] e [12].

A dor ou desconforto torácico é considerado um dos sintomas cardinais das doenças do coração, porém ele pode ser originado de outras estruturas da cavidade torácica. Para avaliar a sua origem, é preciso que o médico use a semiologia para classificar as características da dor, como a qualidade, localização e irradiação, duração, fatores precipitantes ou agravantes e fatores atenuantes [13]. A dor torácica é a expressão sintomatológica de distúrbio cardíaco mais frequente nos serviços de emergência [14].

Dispneia também é uma queixa muito frequente na sala de emergência, sendo motivo de quase 2,5 milhões de atendimentos de urgência nos Estados Unidos, e a definição da causa deste sintoma pode ser cardíaca, principalmente a insuficiência cardíaca congestiva (ICC) ou extracardíaca, como a doença pulmonar obstrutiva crônica (DPOC) [15]. A ICC é a patologia que mais cursa com dispneia e, na maioria dos casos, ela se desenvolve de forma lenta e gradual, por um período de semanas a meses. Em um estudo de coorte de 12.285 pacientes diagnosticados com ICC em um tempo médio de 4,8 anos, a fadiga foi documentada em 4.827 (39\%) deles [13].
A partir da tabela 3, a unidade de emergência do PROCAPE também aborda queixas não cardiológicas, porém esse tipo de paciente pode ser um fator de agravo para o andamento do sistema de atendimento ao serviço de saúde. Os profissionais de saúde de emergência consideraram que pacientes não urgentes diminuem o acesso à emergência para casos reais de emergência, reduzem a qualidade do atendimento, como tempos de espera prolongados, diagnósticos e tratamentos atrasados, atendimento tardio a pacientes gravemente enfermos, e superlotam o sistema [2].

Entretanto, a imprevisibilidade é característica determinante nos serviços de urgência/emergência e muitos dos classificados como atendimento de não emergência poderiam ter chegado com quadro mais grave. Além disso, sintoma é algo pessoal, então muitas queixas podem aparentar gravidade no primeiro momento, mas quando são realizados exames complementares de exclusão observa-se que, na verdade, são situações que poderiam ser controladas de forma ambulatorial [3]. Adicional a isso, cerca da metade a $2 / 3$ dos pacientes com dor torácica internados acabam não confirmando uma causa cardíaca para os seus sintomas, resultando num gasto desnecessário de 5 a 8 bilhões de dólares por ano nos Estados Unidos, mas que denotam difícil controle [16].

Um estudo evidencia que um pequeno grupo de pacientes se 
consideram bem informados sobre o seu estado de saúde e que a grande maioria deles sabe pouco sobre a própria doença (nome e alguns sintomas), mas desconhecem o tratamento e medicamentos indicados [17]. O gráfico 4 mostra uma porcentagem alta em relação àqueles que desconheciam uma cardiopatia $(29,8 \%)$, apenas descobrindo ao serem internados em uma emergência por descompensação clínica. Isso é um fator de agravo à saúde, interferindo nos índices de internações e mortalidade, como também interfere na janela de IAM. Essas internações poderiam ser evitadas na atenção primária à saúde diante de uma atenção resolutiva e no tempo apropriado, sendo as hospitalizações por condições consideradas evitáveis utilizadas como um indicador indireto da efetividade do primeiro nível de atenção à saúde [18]

Ao se analisar os dados do gráfico 5, o resultado foi similar a outro estudo [7,] que de 107 pacientes cardiopatas submetidos à cineangiocoronariografia, $\quad 75,7 \%$ referiram HAS e 37,23\% tinham um IAM prévio.

No Brasil, estima-se que 15 a 20\% da população adulta urbana seja acometida por Hipertensão Arterial Sistêmica. Um estudo de revisão bibliográfica realizou a avaliação clínica dos pacientes que chegam na emergência, e observou que no âmbito das doenças cardiovasculares, destacase a hipertensão arterial, que é a mais prevalente das doenças cardiovasculares e também o principal fator de risco para as complicações mais comuns, como por exemplo acidente vascular cerebral e infarto agudo do miocárdio [19].

Já a síndrome coronariana aguda, que inclui o IAM, é o componente principal da mortalidade cardiovascular do Brasil. Cerca 5 a 15\% dos pacientes atendidos com dor torácica nas salas de emergência norte-americanas têm infarto agudo do miocárdio. Levando para o Brasil, o quantitativo de infartos esperado seria de aproximadamente 400 mil por ano, um número quase 10 vezes maior do que o apresentado pelo SUS. O que se pode supor que grande parte desses pacientes não chegam a tempo ao serviço de emergência [20].

A causa mais comum de dor torácica é a isquemia miocárdica, e a doença arterial coronariana (DAC) é responsável por mais da metade dos eventos cardiovasculares dos pacientes menores de 75 anos [13]. A Organização Pan-Americana de Saúde [21] mostra que apesar das doenças do aparelho circulatório serem a principal causa de óbitos no mundo, elas poderiam ser evitadas com medidas de promoção e prevenção em saúde, assim, diminuindo os riscos e elevando as medidas de diagnóstico e tratamento precoce.

Além disso, há uma elevação nos custos diretos relacionados ao manejo das DAC no Brasil, com alto impacto no orçamento dos órgãos que são financiadores da saúde, especialmente com os gastos em medicamentos, internações e na atenção terciária ou 
quaternária, que são de alta complexidade [22]. Isso denota a importância do auxílio da atenção primária no diagnóstico inicial e acompanhamento clínico dos doentes, além de uma boa orientação quanto a doença que ele apresenta e do controle em hábitos e medicamentos, proporcionando que se diminua o percentual de causas evitáveis que lotem o sistema de emergência.

O perfil clínico dos usuários da emergência do PROCAPE consiste em sua maioria indivíduos do sexo masculino, adultos e idosos, com um quadro clínico cujos sintomas apresentados necessitam do auxílio de um serviço de emergência cardiológica. A sua maior parcela é portadora de uma cardiopatia clínica prévia, concluindo-se que o PROCAPE é um serviço de referência e porta-aberta aos cardiopatas em Pernambuco.

\section{References}

[1] Ribeiro AG, Cotta RMM, Ribeiro SMR. A promoção da saúde e a prevenção integrada dos fatores de risco para doenças cardiovasculares. Cienc Saude Colet 2012; 17(1):7-17.

[2] Durand AC, Palazzolo S, Tantihardouin N, Gerbeaux P, Sambuc R, Gentile S. Nonurgent patients in emergency departments: rational or irresponsible consumers? Perceptions of professionals and patients. BMC Research Notes 2012; 25(5):525.
[3] Coelho, M.F.; Goulart, B.F.; Chaves, L.D.P. Urgências clínicas: perfil de atendimentos hospitalares. Rev Rene 2013; 14(1):50-9.

[4] World Health Organization. Global status report on noncommunicable diseases 2010. Genebra: WHO; 2011. Disponível

em: http:/ / whqlibdoc.who.int/publications /2011/9789240686458_eng.pdf. Acesso em: 30 jan. 2020.

[5] DATASUS [Internet] Brasília: Ministério da Saúde (BR). Departamento de Informática do SUS (DATASUS). Sistema de informação sobre mortalidade (SIM). Disponível em: http: / / www2.datasus.gov.br/DATASU S/index.php?area $=060701$. Acesso em: 21 jan. 2020.

[6] Dawber TR, Moore FE, Mann GV II. Coronary Heart Disease in the Framingham Study. International Journal of Epidemiology 2015; 44(6):1767-1780.

[7] Barbosa $\mathrm{MH}$, Tavares JL, Andrade EV, Silva QCG, Diniz MA, Resende LAPR, Cardoso GL. Aspectos clínicos e epidemiológicos dos clientes submetidos à cineangiocoronariografia. Rev Min Enferm 2011; 15(1):42-46.

[8] Penalva RA, Huoya MO, Correia LCL, Feitosa GS, Ladeia AMT. Perfil lipídico e intensidade de doença aterosclerótica na síndrome coronariana aguda. Arq Bras Cardiol. 2008; 90(1):2430 . 
[9] Santos ES, Timerman A, Baltar VT, Castillo MT, Pereira MP, Minuzzo L, Piegas LS. Escore de risco Dante Pazzanese para síndrome coronariana aguda sem supradesnivelamento do segmento ST. Arq Bras Cardiol 2009; 93(4):343-351.

[10] Schaan BD, Harzheim E, Gus I. Perfil de risco cardíaco no diabetes mellitus e na glicemia de jejum alterada. Rev Saúde Pública 2004; 38(4):529-36.

[11] Cervato AM, Mazzilli RN, Martins IS, Marucci MFN. Dieta habitual e fatores de risco para doenças cardiovasculares. Rev Saúde Pública 1997; 31(3):227-235.

[12] Ferreira SRG, Moura EC, Malta DC, Sarno F. Frequência de hipertensão arterial e fatores associados: Brasil, 2006. Rev Saúde Pública 2009; 43(2):98-106.

[13] Silva RMFL. Semiologia Cardiovascular: Método Clínico, Principais Síndromes e Exames Complementares. 1. ed. Rio de Janeiro: Thieme Revinter Publicações 2009. p. 3669

[14] Lemos DM, Teixeira CSS, Polanczyk CA, Rabelo ER. Trigger points for pain in patients with acute coronary syndrome. Rev Enferm UFSM 2012; 36(3):102-108.

[15] Chircop R, Jelinek GA. B-Type natriuretic Peptide in the Diagnosis of Heart Failure in the Emergency Departament. Emerg Med Aust 2006; 18(2):170-179.
[16] Ewy GA, Ornato JP. 31st Bethesda Conference. Emergency Cardiac Care. J Am Coll Cardiol 2000; 35(4):832-46.

[17] Gomes LC, Fraga MNO. Doenças, hospitalização e ansiedade: uma abordagem em saúde mental. Rev bras enferm 1997; 50(3):425-440.

[18] Campos AZ, Theme-filha MM. Internações por condições sensíveis à atenção primária em Campo Grande, Mato Grosso do Sul, Brasil, 2000 a 2009. Cad Saude Publica 2012; 28(5):845-855.

[19] Bastos FES, Ponte KMA, Sousa JG. Diagnósticos, sinais e sintomas de pacientes em unidade de emergência: estudo bibliográfico. Rev Essentia 2015; 16(2):81-103.

[20] Volschan, A. et al. I Diretriz de Dor Torácica na Sala de Emergência. Arq. Bras. Cardiol 2002; 79(2):1-22.

[21] OPAS. Organização Pan-Americana de Saúde. Organização Mundial de Saúde. Brasil. Doenças cardiovasculares. 2017. Disponível em: https://www.paho.org/bra/index.php ?option=com_content\&view=article\&id $=5253$ : doencas-

cardiovasculares\&Itemid=1096. Acesso em: 04 jul. 2020.

[22] Bezerra GIS, Sena EB, Alves KCG. Premature mortality from diseases of the circulatory system in Palmas, Tocantins. Revista de Patologia do Tocantins 2017; 4(2):54-58.

Conflict of interest: The author declares no conflicts of interest. 
Acknowledgements: None.

Funding: None.

How to cite this article: Figueirêdo

MBM, Borges NB, Souza MBR, Salerno

PR. Perfil clínico dos pacientes no

atendimento médico em uma

emergência cardiológica de referência

no estado de Pernambuco. Brazilian

Journal of Case Reports. 2021 Jul-Sep;

01(3):131-141. 